TITLE: Orientation of Ligand Field for Dangling Manganese in Photosynthetic Oxygen-Evolving Complex of Photosystem II

AUTHORS: Hiroyuki Mino* and Hiroki Nagashima ${ }^{*}$

Division of Material Science, Graduate School of Science, Nagoya University, Furo-cho, Chikusaku, 464-8602, Nagoya, Aichi, Japan.

\title{
Present Addresses
}

${ }^{\ddagger}$ Hiroki Nagashima, Department of Chemistry, Graduate School of Science and Engineering, Saitama University, 255 Shimo-Okubo, Sakura-ku, Saitama 338-8570, Japan

The supporting information containing Figure S1-S8

\section{Corresponding Author}

*Hiroyuki Mino, Tel: +81-52-789-2882; Email: mino@bio.phys.nagoya-u.ac.jp 


\section{CONTENTS}

Figure S1. EPR spectra of $g=4$ signals in $\mathrm{S}_{2}$ state and $\mathrm{S}_{1}$ state of the oriented PS II membranes.

Figure S2. The orientation dependence of the $g=4$ signal at the angle of the external magnetic field relative to the membrane normal $\boldsymbol{n}$.

Figure S3. The simulated spectrum and the energy block diagram for $S=5 / 2$ spin state using the single-spin model.

Figure S4. The possible orientations of the $D_{X} / D_{Y} D_{Z}$ axes relative to the $\mathrm{Mn} 4$ in the crystal structures.

Figure S5. Stereo views of the possible orientations of the $D_{X} / D_{Y} D_{Z}$ axes relative to the ligand fields on $\mathrm{Mn} 1$.

Figure S6. The EPR simulations using the weak coupling model (QM/MM) and the strong coupling models.

Figure S7. $J$ dependence of the resonant conditions for the strong coupled four-spin model.

Figure S8. The simulated $J$ dependence of the resonant fields in the strong coupled model at Q-band $(34 \mathrm{GHz})$ 


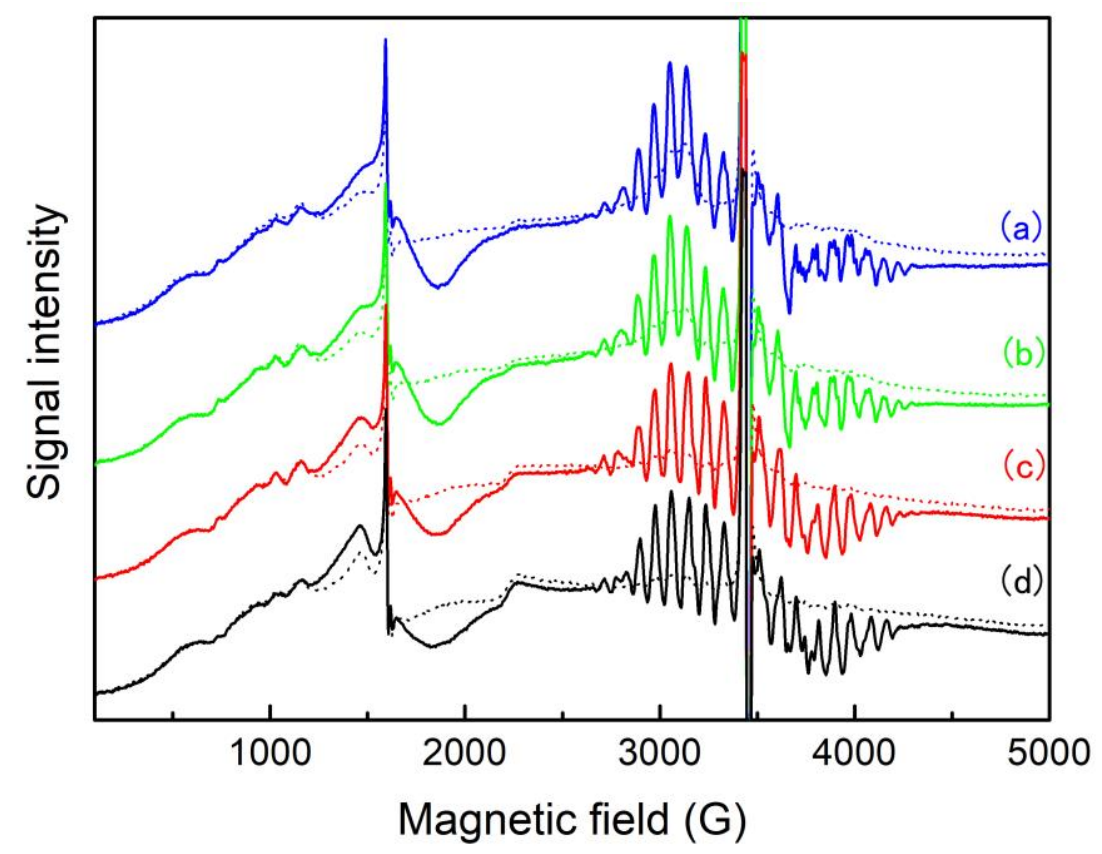

Figure S1. EPR spectra of the oriented PS II membranes in (solid line) $\mathrm{S}_{2}$ state (dotted line) $\mathrm{S}_{1}$ state measured at the angle of (a) $0^{\circ}$, (b) $30^{\circ}$, (c) $60^{\circ}$, and (d) $90^{\circ}$ of the external magnetic field $\boldsymbol{B}_{0}$ relative to the membrane normal $\boldsymbol{n}$. Experimental conditions: microwave frequency, $9.67 \mathrm{GHz}$; microwave power, $4 \mathrm{~mW}$; modulation frequency, $100 \mathrm{kHz}$; modulation amplitude, $10 \mathrm{G}$.

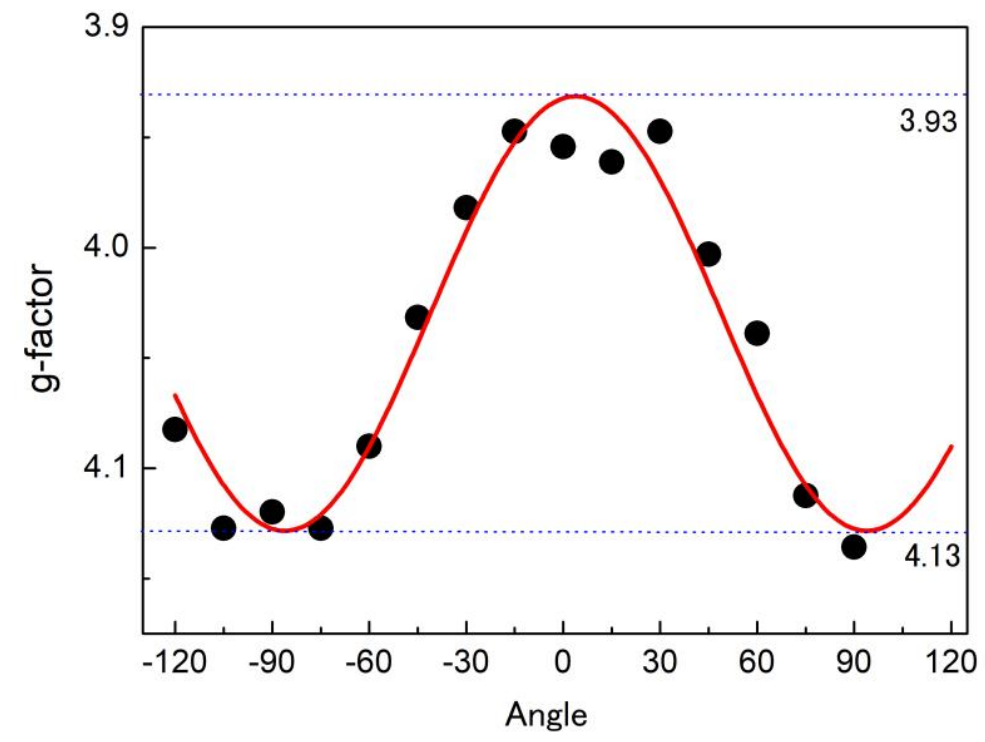

Figure S2. The orientation dependence of the $g$-values, converted from the resonant fields of the $g=$ 4.1 signal relative to the angle between the external magnetic field $\boldsymbol{B}_{\boldsymbol{0}}$ and the membrane normal $\boldsymbol{n}$. The orientation dependence was fitted by a sine function (solid line). The dotted lines show the maximum and minimum $g$-values. Experimental conditions are the same as fig. S1. 

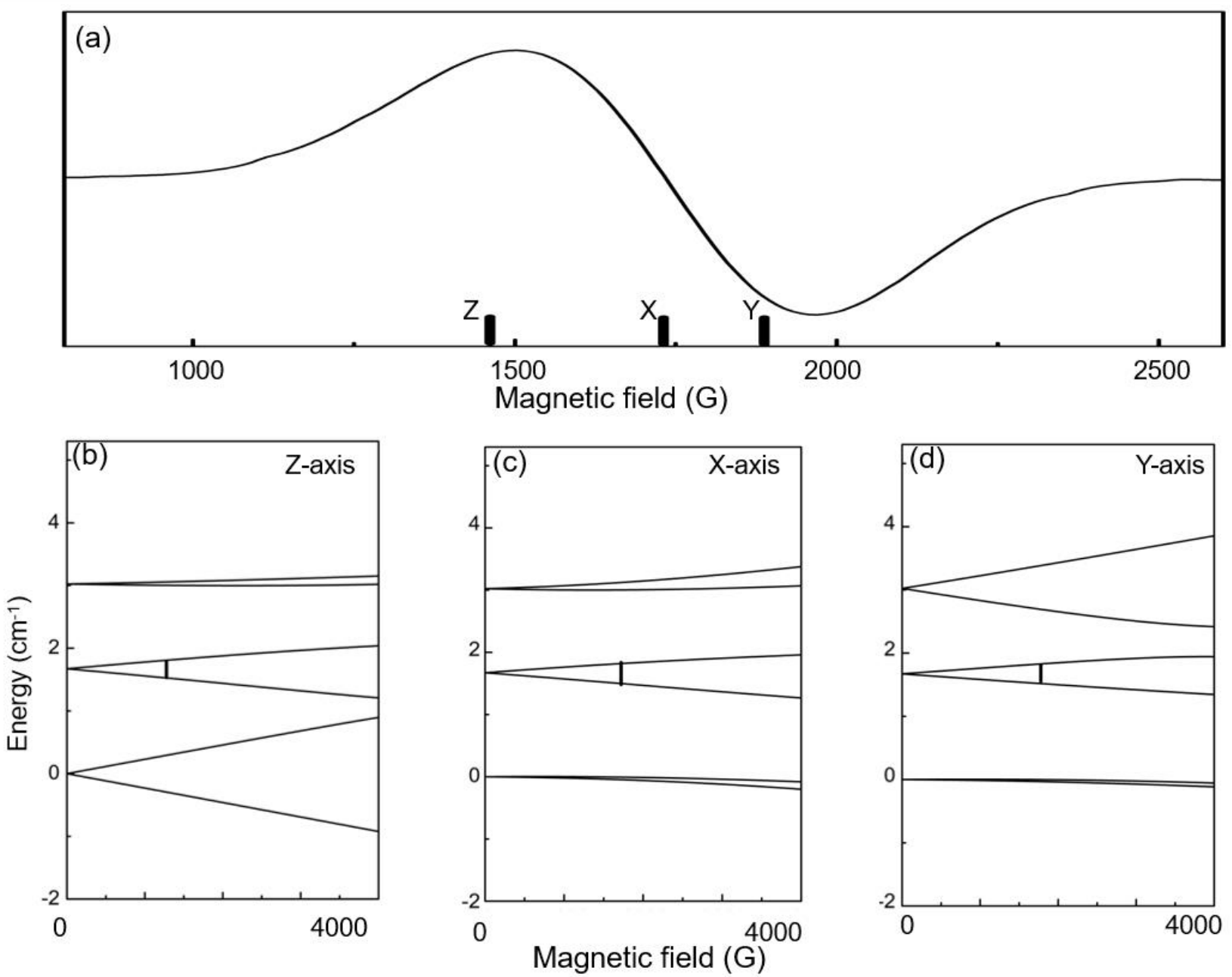

Figure S3. (a) The powder pattern of the EPR spectrum for the $g=4.1$ signal using the single-spin model. (b-d) The energy block diagrams, where the external field $\boldsymbol{B}_{\mathbf{0}}$ is directed along (b) $D_{Z}$-axis, (c)

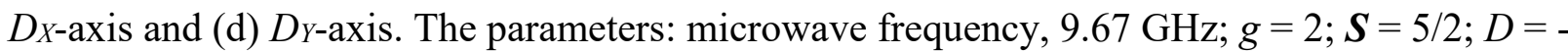
$0.455 \mathrm{~cm}^{-1} ; E / D=0.25$. 

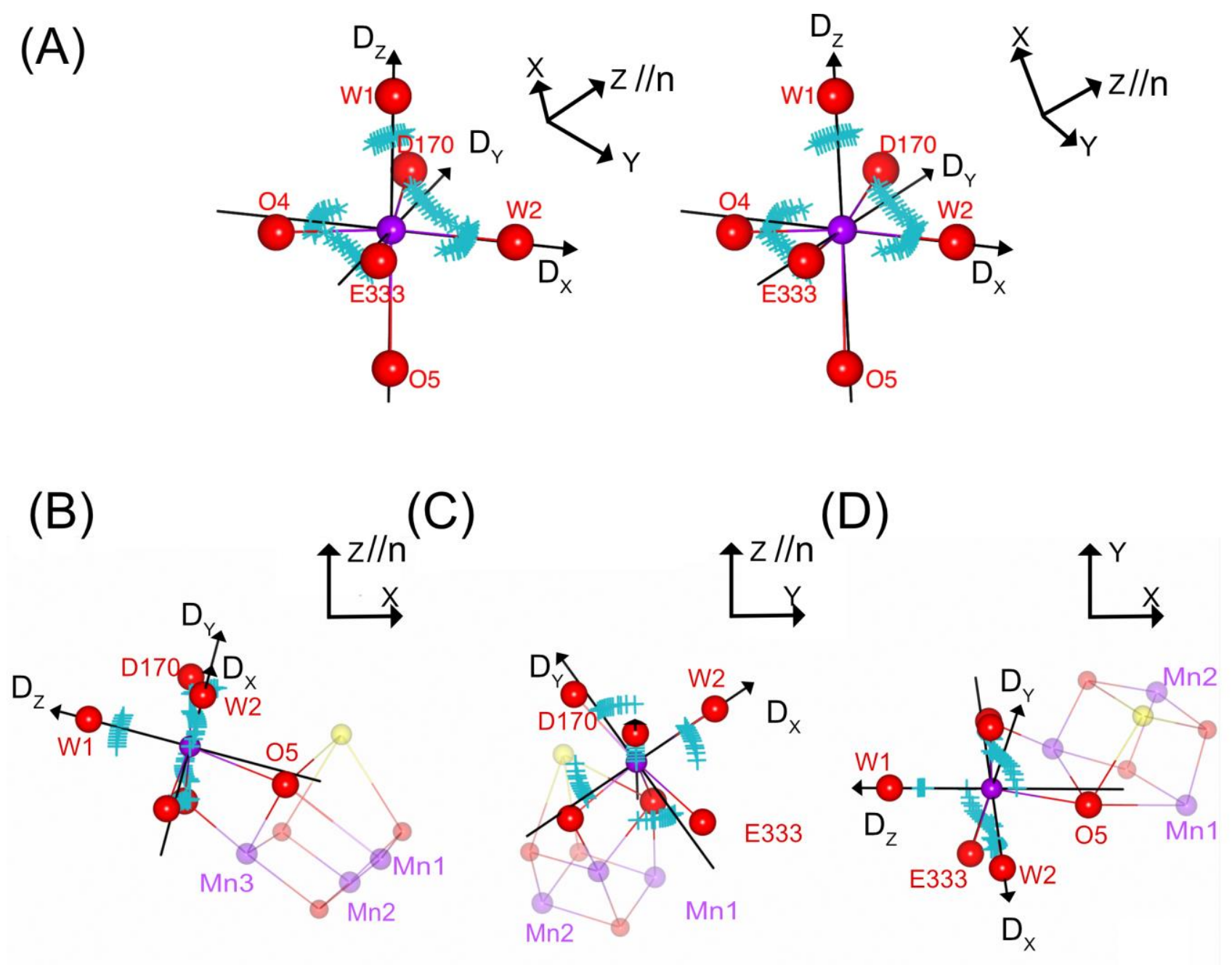

Figure S4. The possible orientations of the $D_{X} / D_{Y} D_{Z}$ axes relative to the $\mathrm{Mn} 4$ in the crystal structures. The Mn4 is located on the origin of the coordinates. The Z-axis is parallel to membrane normal $\boldsymbol{n}$. The XY-plane is parallel to membrane plane. $D_{X}$ and $D_{Z}$ axes are set to the closest direction to $\mathrm{Mn} 4-\mathrm{O}(\mathrm{W} 2)$ and $\mathrm{Mn} 4-\mathrm{O}(\mathrm{W} 1)$, respectively. Panel A is stereographic figure, projected to the arbitrary plane. Panels B-D are the projected figures to (B) XZ-, (C) YZ- and (D) XY-planes, respectively. The possible angles for EPR shifts of 70-80 $\mathrm{G}$ in the oriented membranes are indicated with the blue cross marks, corresponded to the open circles in fig.3. The red, yellow and purple balls represent $\mathrm{O}, \mathrm{Ca}$ and $\mathrm{Mn}$, respectively. 

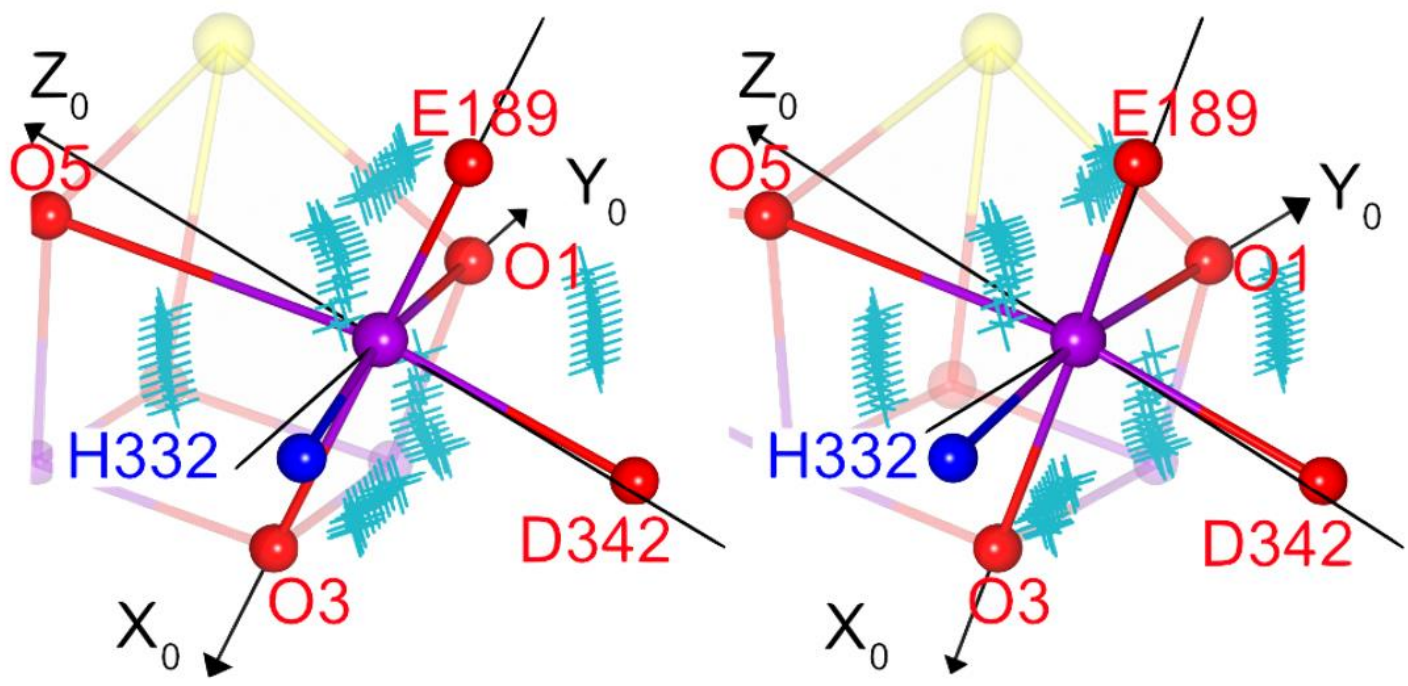

Figure S5. Stereo views of the orientations of the $X_{0} / Y_{0} / Z_{0}$ axes relative to the ligand fields on $\mathrm{Mn} 1$. The Mn1 is located on the origin of the coordinates. The axes are set close to Mn1-O3, Mn1-O1 and Mn1-O5, respectively. The possible angles for EPR shifts of 70-80 G in the oriented membranes are indicated with the blue cross marks, corresponded to the open circles in fig.3. The red, yellow and purple balls represent $\mathrm{O}, \mathrm{Ca}$ and $\mathrm{Mn}$, respectively. 


\section{Four-spin model}

For the four coupled manganese model, Hamiltonian is written as followings:

$$
\begin{gathered}
\mathcal{H}=\sum_{i=1}^{4} g_{i} \beta \boldsymbol{S}_{\boldsymbol{i}} \boldsymbol{B}_{\mathbf{0}}+\sum_{\boldsymbol{i}} \cdot \boldsymbol{A}_{\boldsymbol{i}} \cdot \boldsymbol{S}_{\boldsymbol{i}}+\sum_{i=1}^{4} \boldsymbol{S}_{\boldsymbol{i}} \cdot \boldsymbol{D}_{\boldsymbol{i}} \cdot \boldsymbol{S}_{\boldsymbol{i}}-\sum_{i<j} 2 J_{i j} \boldsymbol{S}_{\boldsymbol{i}} \cdot \boldsymbol{S}_{\boldsymbol{j}} \\
\boldsymbol{S}_{\text {total }}=\sum_{i=1}^{4} \boldsymbol{S}_{\boldsymbol{i}}
\end{gathered}
$$

, where $\boldsymbol{S}_{\boldsymbol{i}}$ and $\boldsymbol{I}_{i}$ are the operators of electron spin and nuclear spin of the $i$-th Mn ion, respectively, $g_{i}$ is the $g$-factor, and $\boldsymbol{A}_{\boldsymbol{i}}$ is the effective hyperfine tensor of the $i$-th ion, $\boldsymbol{D}_{\mathrm{i}}$ is the tensor of zero-field splittings. $J_{i j}$ is the exchange interaction between the $i$-th and $j$-th ions. Total spin $\boldsymbol{S}_{\text {total }}=13 / 2$ for $1 \mathrm{Mn}(\mathrm{III}) 3 \mathrm{Mn}(\mathrm{IV})$. For two-spin model, the coupling $J_{\text {eff }}$ was assumed between $\boldsymbol{S}=9 / 2$ (cubane frame) and $\boldsymbol{S}=2(\mathrm{Mn}(\mathrm{III}))$. For four-spin model, the spectral simulations were performed by the diagonalization of the $320 \times 320$ matrix for spin Hamiltonian using basic set of wavefunctions $\left(\left(\left(\boldsymbol{S}_{1} \otimes \boldsymbol{S}_{2}\right) \otimes \boldsymbol{S}_{3}\right) \otimes \boldsymbol{S}_{4}\right)$. In the simulations, Mn1-3 were assumed to be $\operatorname{Mn}(\mathrm{IV})(\boldsymbol{S}=3 / 2)$, and Mn4 was assumed to be $\operatorname{Mn}(\mathrm{III})(\boldsymbol{S}=2)$. The zero-field splitting term was replaced as followings ${ }^{1}$ :

$$
d_{4}\left[\boldsymbol{S}_{4, \mathbf{z}}^{2}-\frac{1}{3} S_{4}\left(S_{4}+1\right)\right]+e_{4}\left(\boldsymbol{S}_{4, \boldsymbol{x}}^{2}-\boldsymbol{S}_{4, \boldsymbol{y}}^{2}\right)
$$

, where $d_{4}$ and $e_{4}$ are onsite zero-field splitting parameters for Mn4. For the weak coupling (QM/MM) model, the set of $J$ couplings is following: $J_{12}: 30.5 \mathrm{~cm}^{-1}, J_{13}: 13.0 \mathrm{~cm}^{-1}, J_{14}: 0 \mathrm{~cm}^{-1}, J_{23}$ : $35.5 \mathrm{~cm}^{-1}, J_{24}: 0 \mathrm{~cm}^{-1}, J_{34}:-7.6 \mathrm{~cm}^{-1} 2$. For the strong coupling models, the set of $J$ couplings is following: $J_{12}: 200 \mathrm{~cm}^{-1}, J_{13}: 0$ or $200 \mathrm{~cm}^{-1}, J_{14}: 0 \mathrm{~cm}^{-1} ; J_{23}: 200 \mathrm{~cm}^{-1} ; J_{24}: 0 \mathrm{~cm}^{-1}$. If $J_{14}$ is non-zero, the result is almost the same because the cubane frame is under the strong ferromagnetic couplings. Figure S6 shows the comparison of (a) the QM/MM (weak coupling) model, (b, c) two-spin model and (d-g) the strong coupling models. In (a) the QM/MM model, the low field signal was assigned to the high spin state as $g=6$ and $g=10$, and the high field signal was upshifted from the $g=4$ by mixing of the weakly excited state. In order to simulate the spectrum of the $g=4$ signal for the strong coupling models, larger $\left|J_{34}\right|$, estimated as $>\sim 30 \mathrm{~cm}^{-1}$, is required. The $g=4$ signal was well reproduced using the onsite zero-field splitting $d_{4}=-3$ to -2 with the strong $J_{34}$ coupling. 


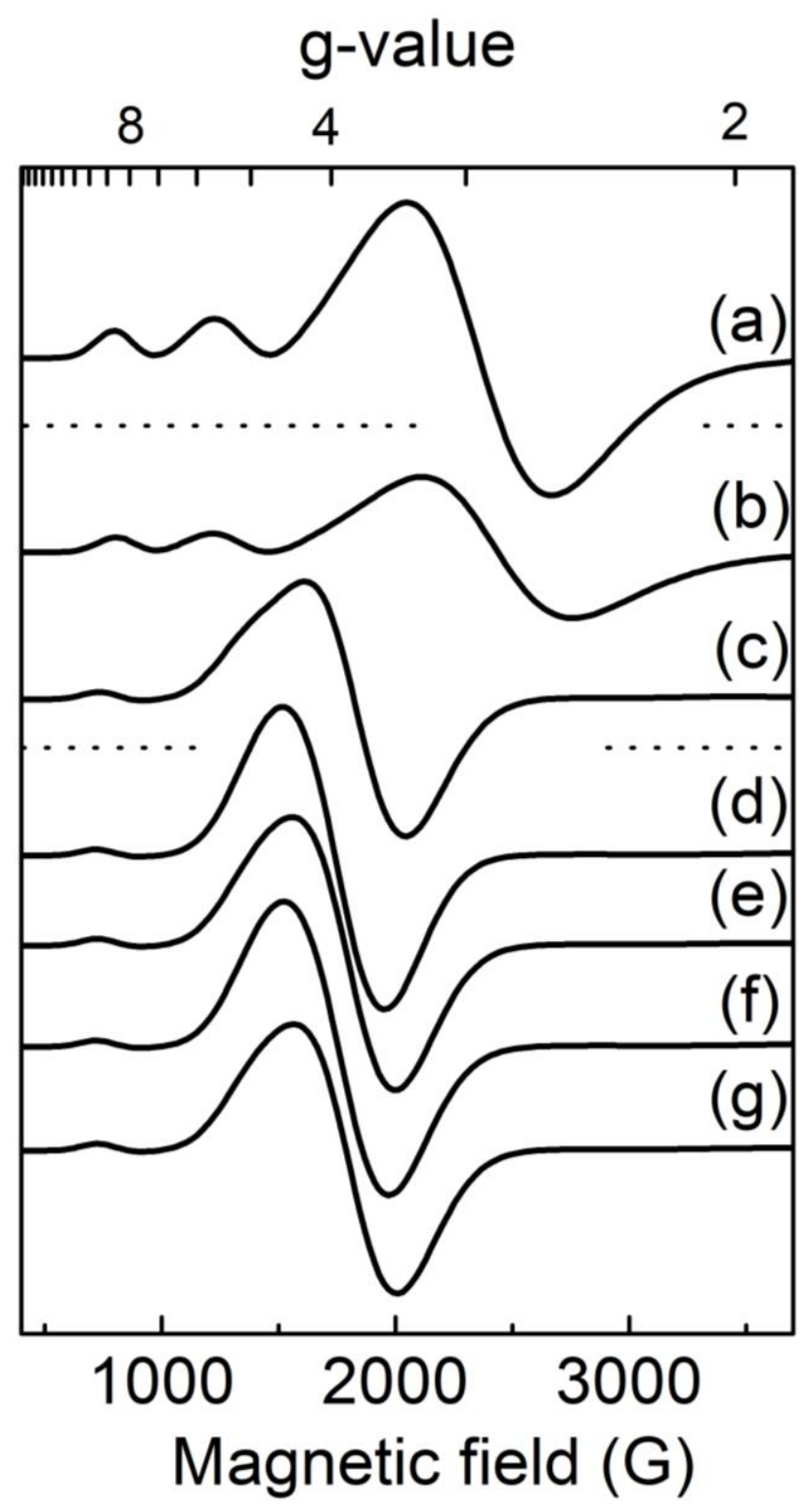

Figure S6. The EPR simulations using (a) the weak coupling model (QM/MM), (b, c) two-spin model and (d-g) the strong coupling models. The exchange couplings in four-spin models $\left[J_{12}, J_{13}\right.$, $\left.J_{14}, J_{23}, J_{24}, J_{34}\right]:$ (a) $[30.5,13,0,35.5,0,-7.6]$, (d) [200 ,200, 0, $\left.200,0,-200\right]$, (e) [200 , 0, 0, 200, $0,-50]$, (f) $[200,0,0,200,0,-200]$, (g) $[200,0,0,200,0,-50]$. The exchange couplings $J_{\text {eff }}$ in twospin model: (b) $J_{\text {eff }}=-2.3 \mathrm{~cm}^{-1}$, (c) $J_{\text {eff }}=-10 \mathrm{~cm}^{-1}$. (a-c) $d 4=-3 \mathrm{~cm}^{-1}$, (d-g) $d 4=-2.3 \mathrm{~cm}^{-1}, e 4 / d 4=$ 0.25. Microwave frequency $9.67 \mathrm{GHz}$; Gaussian linewidth, $350 \mathrm{G}$. 

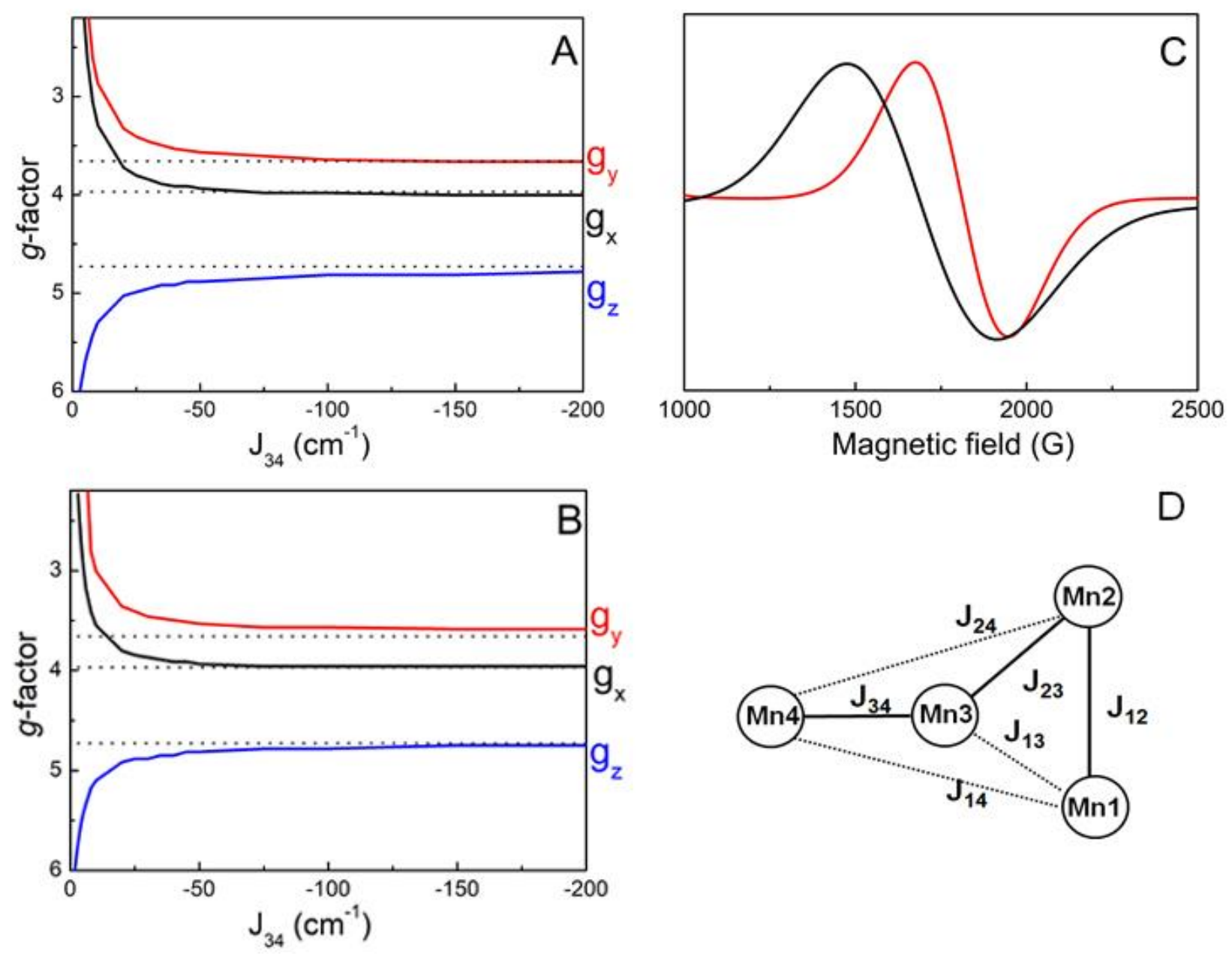

Figure S7. (A, B) $J$ dependence of the resonant conditions for the strongly coupled four-spin model. The parameters: $J_{12}: 200 \mathrm{~cm}^{-1}, J_{13}: 0 \mathrm{~cm}^{-1}, J_{14}: 0 \mathrm{~cm}^{-1}, J_{23}: 200 \mathrm{~cm}^{-1}, J_{24}: 0 \mathrm{~cm}^{-1}$. (A): $d 4,-2 \mathrm{~cm}^{-1}$; $e 4 / d 4=0.25$. (B): $d 4,-3 \mathrm{~cm}^{-1} ; e 4 / d 4=0.25$. The solid lines show the resonant fields along each axis. The dotted lines show the resonant condition in the case of the single-spin model for $S=5 / 2$ (Figure S3). (C) The simulated spectra for the oriented sample at (red) $0^{\circ}$ and (black) $90^{\circ}$ using the parameters of $d 4=-2.3 \mathrm{~cm}^{-1}, e 4 / d 4=0.25$ and $J_{34}=-50 \mathrm{~cm}^{-1}$. The other conditions are the same as the simulated spectrum in the single-spin model. (D) The scheme for the set of the exchange couplings for the four-spin model. Microwave frequency, $9.67 \mathrm{GHz}$. 
Haddy et al. observed the $g=3.14$ and 4.6 signals in Q-band EPR ${ }^{3}$. The $g=3.14$ signal was assigned to the $\pm 3 / 2$ transition along the $x$-axis for $S=5 / 2$. The $g=4.6$ signal was tentatively assigned to another transitions within $S=5 / 2^{3}$. The Q-band spectrum was well reproduced using the onsite zero-field splitting $\mathrm{d} 4=-2.3 \mathrm{~cm}^{-1}$ in the strong coupling model (Figure $\mathrm{S} 8$ ).
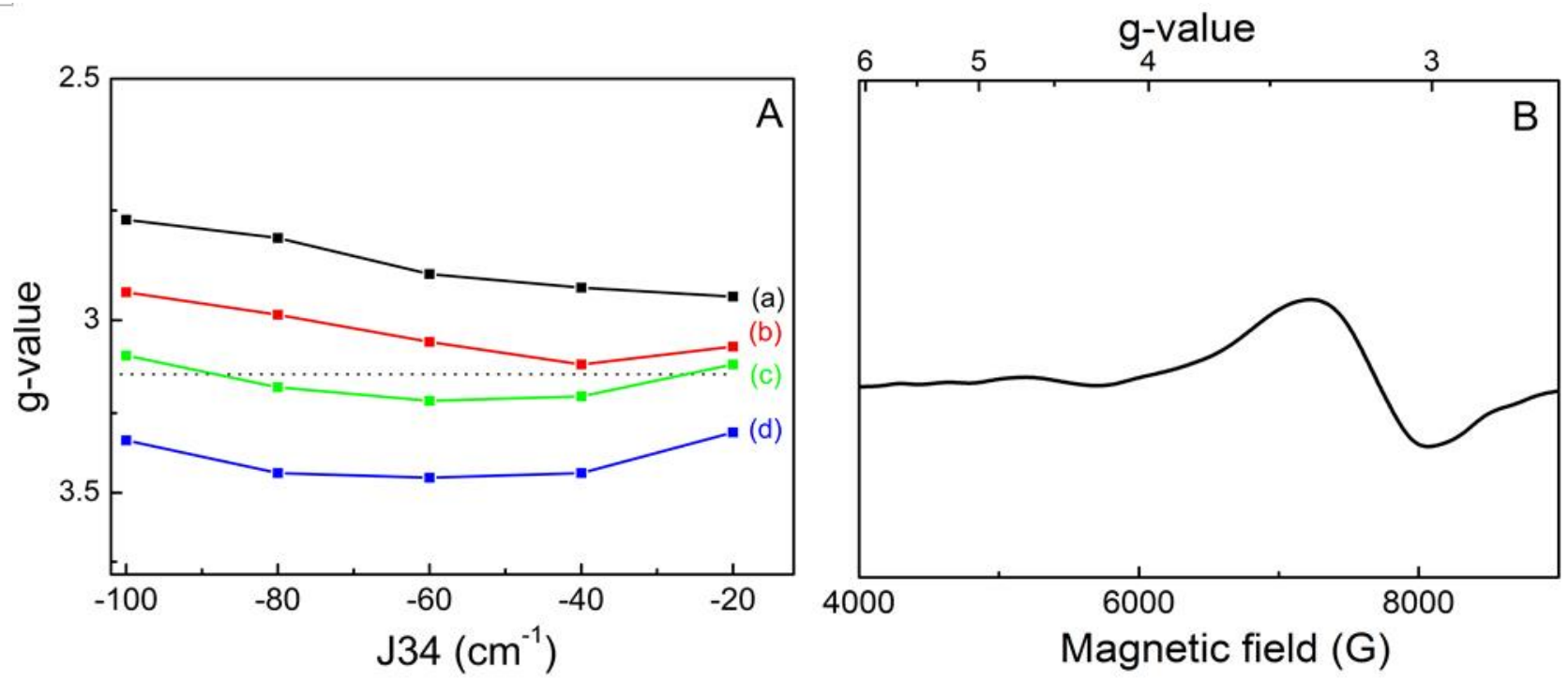

Figure S8. (A) The simulated $J$ dependence of the resonant fields in the strong coupled model at Qband (34 GHz). The parameter set was used as: $J_{12}: 200 \mathrm{~cm}^{-1}, J_{13}: 0 \mathrm{~cm}^{-1}, J_{14}: 0 \mathrm{~cm}^{-1}, J_{23}: 200 \mathrm{~cm}^{-1}$, $J_{24}: 0 \mathrm{~cm}^{-1}$, and $e 4 / d 4=0.25$; (a) $d 4,-2.0 \mathrm{~cm}^{-1}$; (b) $d 4,-2.2 \mathrm{~cm}^{-1}$; (c) $d 4,-2.4 \mathrm{~cm}^{-1}$; (d) $d 4,-3 \mathrm{~cm}^{-1}$. The dotted line indicates $g=3.14^{3}$. (B) The simulated EPR spectrum using $d_{4}=-2.3 \mathrm{~cm}^{-1}$. The parameters are the same as (A) except for $J_{34}=-50 \mathrm{~cm}^{-1}$. 


\section{References}

(1) Cox, N.; Ames, W.; Epel, B.; Kulik, L. V.; Rapatskiy, L.; Neese, F.; Messinger, J.; Wieghardt, K.; Lubitz, W. Electronic Structure of a Weakly Antiferromagnetically Coupled $\mathrm{Mn}^{\mathrm{II}} \mathrm{Mn}^{\mathrm{III}}$ Model Relevant to Manganese Proteins: A Combined EPR, ${ }^{55} \mathrm{Mn}-\mathrm{ENDOR}$, and DFT Study. Inorg. Chem. 2011, 50, 8238-8251.

(2) Pantazis, D. A.; Ames, W.; Cox, N.; Lubitz, W.; Neese, F. Two interconvertible structures that explain the spectroscopic properties of the oxygen-evolving complex of photosystem II in the $\mathrm{S}_{2}$ state. Angew. Chem., Int. Ed. 2012, 51, 9935-9940.

(3) Haddy, A.; Lakshmi, K. V.; Brudvig, G. W.; Frank, H. A. Q-band EPR of the $\mathrm{S}_{2}$ state of Photosystem II confirms an $\mathrm{S}=5 / 2$ origin of the X-band $g=4.1$ signal. Biophys. J. 2004, 87, 28852896. 